\title{
La scolarité obligatoire en Algérie : ambitions et défis
}

Compulsory schooling in Algeria: Ambitions and challenges

La educación obligatoria en Argelia: ambiciones y desafíos

\section{Mina Tounsi}

\section{(2) OpenEdition}

\section{Journals}

Édition électronique

URL : https://journals.openedition.org/ries/5601

DOI : 10.4000/ries.5601

ISSN : 2261-4265

Éditeur

France Education international

Édition imprimée

Date de publication : 1 décembre 2016

Pagination : 47-56

ISBN : 978-2-85420-612-8

ISSN : $1254-4590$

\section{Référence électronique}

Mina Tounsi, «La scolarité obligatoire en Algérie : ambitions et défis », Revue internationale d'éducation de Sèvres [En ligne], 73 | décembre 2016, mis en ligne le 01 décembre 2018, consulté le 01 juillet 2021. URL : http://journals.openedition.org/ries/5601; DOI : https://doi.org/10.4000/ries.5601 


\title{
La scolarité obligatoire en Algérie : ambitions et défis
}

\author{
Mina Tounsi \\ Inspectrice de l'éducation honoraire, Algérie
}

La rentrée scolaire 2016-2017, annonciatrice de nouveaux programmes est propice à une réflexion sur ce que l'école enseigne à tous dans le cadre de la scolarité obligatoire en Algérie. Avec la définition des Objectifs de développement durable $^{1}$ de l'ONU (ODD), après la fin des Objectifs de l'éducation pour tous (EPT) et les Objectifs de développement du millénaire (OMD), en 2015, il semble essentiel de s'interroger sur ce que signifie cette obligation en termes d'apprentissage ainsi que sur les contenus de cet enseignement obligatoire. Est-il pensé comme commun à tous les élèves ? L'école algérienne valorise-t-elle un projet spécifique d'éducation correspondant à des choix ? Lesquels ? S'agit-il de choix individuels ou collectifs?

Pour tenter d'apporter quelques réponses à ces questions, nous présentons, dans un premier temps, les finalités, la structure et l'organisation du système éducatif algérien en situant la politique éducative conduite par rapport à d'autres systèmes éducatifs. Puis, après avoir exposé le cadre conceptuel des nouveaux programmes et les champs disciplinaires, inscrits dans une perspective curriculaire, nous formulons quelques propositions pour aider à concrétiser l'ouverture et l'intégration de l'école algérienne au mouvement universel de progrès.

En Algérie, l'enseignement obligatoire se déclare inclusif en stipulant, dans la Loi d'orientation sur l'éducation nationale de 2008, article 14, le droit à l'enseignement et l'intégration scolaire des enfants à besoins spécifiques (dont la durée de scolarisation peut être prolongée de deux ans). Il s'adresse surtout à une population scolaire hétérogène dont il est soucieux de "prendre en charge les besoins, en plus des apprentissages nécessaires à tous, en offrant les souplesses indispensables pour respecter la diversité des demandes, des attentes, des rythmes de développement. » (Commission nationale des programmes, 2009).

Ces principes relèvent de l'équité et de l'égalité des chances. D'autre part, l'enseignement de base ou enseignement fondamental doit assurer un enseignement commun à tous les élèves, leur permettant :

- d'acquérir les savoirs fondamentaux nécessaires et les compétences essentielles ;

1. Recommandations d'Irina Bokova, directrice générale de l'Unesco, à la conférence « Éducation en vue du développement durable et rôle du Programme 2030 ", Suède, 7 juillet 2016, ODD 4 pour l'éducation. 
- soit la poursuite de leur scolarité dans le niveau d'enseignement suivant, soit leur intégration dans l'enseignement et la formation professionnels, soit la participation à la vie de la société. ${ }^{2}$

On peut se demander si cet enseignement obligatoire est réellement pensé comme commun à tous les élèves, s'il valorise leurs capacités, s’il oriente judicieusement des millions d'élèves, s’il est coordonné adéquatement avec la formation et l'enseignement professionnels, s'il remplit efficacement sa mission de «mur porteur» dans la société.

Pour atteindre cette finalité d'un enseignement obligatoire conçu comme commun pour la formation «d'un citoyen doté de repères nationaux incontestables, profondément attaché aux valeurs du peuple algérien, capable de comprendre le monde qui l'entoure, de s'y adapter et d'agir sur lui et en mesure de s'ouvrir sur la civilisation universelle $»^{3}$, le système scolaire algérien a connu, à la faveur de la réforme de l'éducation, en 2003, une avancée juridique notable, avec, d'une part, l'affirmation du caractère obligatoire de l'enseignement pour toutes les filles et tous les garçons âgés de 6 à 16 ans révolus, assorti de sanctions à l'encontre des personnes responsables de manquements à cette disposition et, d'autre part, une restructuration, avec la mise en place :

- de l'éducation préscolaire pour les enfants de 5 ans (et dont on espérait la généralisation à tout le territoire national, prévue en 2008) ;

- de l'enseignement fondamental aménagé (enseignement primaire réduit de 6 à 5 années et enseignement moyen prolongé de 3 à 4 années);

- d'un enseignement secondaire général et technologique offrant des parcours diversifiés.

Enfin, des mesures d'importance majeure ont été prises concernant l'élaboration des programmes, l'approbation et l'homologation des manuels scolaires et l'accès aux nouvelles technologies. En outre, la création d'établissements privés d'éducation et d'enseignement agréés par le ministère de l'éducation nationale (MEN) a représenté une ouverture.

Les aménagements de la durée des cycles (préservant la durée globale de 9 ans), et la mise en place d'un système d'évaluation formative, visent essentiellement à permettre à un maximum d'élèves d'atteindre les objectifs de l'enseignement de base.

\section{LES POURVOYEURS DE SAVOIRS FONDAMENTAUX}

Le système éducatif algérien est national et postule une même éducation pour tous. L'enseignement est majoritairement public, avec une faible représentation des écoles privées relevant du MEN. L'article 18 de la Loi d'orientation de 2008, qui définit la vocation de l'école algérienne, dispose que «l'éducation

2. Loi d'orientation sur l'éducation nationale de 2008 , article 44 .

3. Ibid., article 2. 
nationale repose sur le secteur public, mais la possibilité de créer des établissements privés d'éducation et d'enseignement peut être accordée aux personnes physiques ou morales de droit privé. » Cette " possibilité » venue consacrer une pratique informelle datant de plus de quinze ans, à l'initiative de quelques familles et associations, a permis l'exercice d'un enseignement privé réglementé. Aujourd'hui, parallèlement au secteur public (18441 écoles primaires et 5252 collèges $)^{4}, 320$ écoles privées, implantées dans les grandes villes, dispensent un enseignement notamment dans le cadre de la scolarité obligatoire.

Au début, la plupart de ces écoles dispensait un enseignement en langue française. Cependant, les écoles privées étant tenues par la loi de dispenser l'enseignement en langue arabe, langue nationale officielle, et d'appliquer les programmes nationaux, elles ont dû s'adapter, d'autant que la scolarité des élèves de ces établissements est sanctionnée par les mêmes examens que ceux du secteur public. Si quelques écoles «offrent » aux élèves un double programme, ou plutôt une double langue d'enseignement, cette pratique non généralisée - d'ailleurs dénoncée par l'Association nationale des écoles privées agréées (Anepa) pour des raisons pédagogiques - ne fait pas l'unanimité dans la société.

Aujourd'hui, le MEN estime que les écoles privées, qui scolarisent 150000 élèves, un effectif très éloigné des 8,5 millions d'élèves dans le secteur public (dont les deux tiers dans l'enseignement obligatoire), ont une obligation de service public. Cette reconnaissance s'exprime dans l'intérêt porté à leurs offres de services (activités périscolaires, projets pédagogiques, etc.) et dans la volonté de les impliquer dans le processus d'amélioration de la qualité de l'enseignement par la participation aux débats sur l'éducation ${ }^{5}$. La tutelle exige aussi que leur encadrement pédagogique (essentiellement des retraités de l'éducation nationale) soit formé régulièrement, et offre même une participation aux sessions organisées par le MEN.

La tendance est donc à l'établissement d'un partenariat, pour répondre à des normes et standards nationaux et internationaux : nombre d'élèves par classe, manuel scolaire/élève, pourcentage de réussite aux examens. Sur ce dernier point, les résultats ne sont pas significatifs en dépit des résultats affichés par les écoles privées : $100 \%$ de réussite à l'examen de passage de $5^{\mathrm{e}}$ année du primaire et entre 60 et $100 \%$ au brevet d'enseignement moyen (BEM), du fait de la place marginale de l'enseignement privé (1\% des effectifs scolarisés).

Les prestations supplémentaires (cours d'informatique, terminologie, préparation au brevet et au baccalauréat français) de ces écoles n'empêchent pas le développement du phénomène paradoxal des cours particuliers payants, dès le cycle primaire. Des études permettraient d'indiquer s'il s'agit d'un effet de mode ou d'une compétition sociale significative d'une forme d'élitisme.

4. Enquête statistique, 2015, Ministère de l'éducation nationale.

5. Conférence nationale d'évaluation de la réforme à mi-parcours (2014), Assises nationales sur l'éducation (2015) et représentation à PISA 2015. 


\section{LES TROIS PÉRIODES \\ DU SYSTÈME ÉDUCATIF ALGÉRIEN}

\section{Une école accessible à tous}

Consciente des enjeux en matière d'éducation, l'Algérie s'est, au lendemain de son indépendance (1962) et jusqu'à fin des années 1970, attelée à faire fonctionner les écoles, les équipes administratives et pédagogiques, à distribuer les manuels scolaires dans tout le pays. Parallèlement et progressivement, un enseignement en langue arabe a été mis en place. L'école est gratuite et obligatoire. Les taux croissants de scolarisation permettent de rattraper un énorme retard et d'effacer les séquelles de la période coloniale.

L'évolution du taux de scolarisation de la tranche d'âge des six ans, qui est actuellement de $97 \%$, alors qu'il n'était que de 43,5\% en 1965, est un indice révélateur des résultats obtenus en matière de scolarisation. (Préambule de la Loi d'orientation de 2008)

\section{La recherche d'un modèle d'éducation pour tous}

De 1980 jusqu'à fin des années 1990, l'enseignement fondamental et polytechnique concrétise l'arabisation des enseignements et l'algérianisation de l'encadrement à tous les niveaux. La langue arabe est langue d'enseignement. Mais la formation initiale des enseignants du collège, assurée dans les instituts de technologie de l'éducation, avec pour seul critère d'entrée un niveau de classe terminale, affectera sensiblement la qualité des enseignements et le rendement du système dans son ensemble. Ce recrutement massif est opéré alors même que de nouvelles matières (technologie et informatique) sont introduites.

Sur un autre plan, cependant, l'Institut national de recherche en éducation (INRE) développe dès 1996 des procédures d'évaluation des programmes sur la base de critères et d'indicateurs de qualité.

\section{La meilleure éducation pour chacun}

Au début des années 2000, l'Algérie passe à une nouvelle optique de rentabilité pédagogique. Les efforts portent sur la qualité des apprentissages et l'accroissement du rendement du système éducatif. Dans un contexte d'avènement du pluralisme politique et de mondialisation, et sur la base des principes fondateurs de la nation algérienne - islamité, arabité, amazighité, dont l'intégration constitue l'algérianité -, une réforme du secteur est engagée. De nouveaux dispositifs pédagogiques vont consacrer trois types d'articulations (Commission nationale des programmes, 2009) :

- la détermination d'un socle commun de compétences, dont doivent être dotés tous les jeunes, à l'issue de l'école de base ; 
- l'ajustement, l'adaptation et le redéploiement d'un enseignement plus proche des impératifs de la vie réelle que des considérations administratives et bureaucratiques ;

- l'amélioration qualitative des résultats scolaires comme axe prioritaire des nouvelles missions de l'école algérienne : instruire, socialiser et qualifier.

\section{LE CADRE CONCEPTUEL DES NOUVEAUX PROGRAMMES}

Les programmes, clé de voûte de l'amélioration qualitative, ont aussi une histoire. Aux trois périodes du système éducatif algérien post indépendance correspondent trois entrées dans les programmes : d'abord une entrée par les contenus disciplinaires enseignés dans une transmission frontale, du maître vers l'élève. Puis l'approche par objectifs a donné aux programmes un caractère structuré et permis de dessiner des profils d'élèves. Enfin, une logique d'apprentissage centrée sur l'élève, inspirée par la théorie du socioconstructivisme, a été privilégiée. On s'oriente vers l'approche par compétences ${ }^{6}$, vu l'immensité des champs de la connaissance. Afin d'amener les élèves vers des connaissances « utiles » par la maîtrise de sujets divers, les groupes spécialisés de discipline conçoivent les programmes de "première génération », appliqués en 2003.

Dans le cadrage de la Loi d'orientation, la Commission nationale des programmes s'est dotée d'un Référentiel général des programmes (RGP), publié en 2009, définissant, en amont les fondements des programmes et leur ancrage national et universel, le cadre des finalités et les concepts opératoires, le domaine des savoirs à faire acquérir et leur organisation dans les champs disciplinaires ainsi que les dispositifs d'évaluation. Élaborés à partir de choix axiologiques (les valeurs sont le substrat des programmes), épistémologiques (les contenus d'enseignement s'organisent autour des concepts « structurants » de la discipline) et méthodologiques (l'approche curriculaire, qui donne pratiquement une autre mission aux disciplines), les programmes veulent développer chez l'élève des connaissances, des compétences et des valeurs.

Objets d'allègements (parfois arbitraires) en 2008/2009, d'évaluations nationales en 2014-2015 et d'ajustements, ils aboutissent aux programmes de deuxième génération. Le concept de curriculum, défini dans le RGP, prend alors tout son sens :

Le concept de curriculum « désigne toutes les expériences d'apprentissage organisées, l'ensemble des influences auxquelles pourrait être exposé l'élève sous la responsabilité de l'école pendant une période de formation. Ce concept englobe les activités d'apprentissage auxquelles l'élève participe, les méthodes et les moyens utilisés ainsi que les procédures d'évaluation retenues.

6. «Doter les élèves de compétences pertinentes, solides et durables susceptibles d'être exploitées à bon escient dans des situations authentiques de communication et de résolution de problèmes et qui les rendent aptes à apprendre toute leur vie, à prendre une part active dans la vie sociale, culturelle et économique et à s'adapter aux changements ", Loi d'orientation de 2008 , article 4. 
En vigueur en $2016^{7}$, ces programmes sont marqués par une meilleure cohérence verticale (différents niveaux d'enseignement) et horizontale (diverses disciplines), ainsi que par une plus grande visibilité des valeurs républicaines, identitaires, sociales relatives aux options nationales et aux valeurs universelles ; ils apporteront une solution pédagogique aux dysfonctionnements de l'école. C'est dans une logique de cycles puis de paliers que se déroulent les apprentissages organisés dans les champs disciplinaires.

\section{Les champs disciplinaires}

L'enseignement obligatoire, qui repose sur le principe de la priorité donnée à l'élève, vise, dans l'enseignement primaire, à développer des compétences de base dans les domaines de l'expression orale et écrite, de la lecture, des mathématiques, des sciences, de la morale civique et religieuse (prise de conscience du temps, de l'espace, des objets et de son propre corps), tout en développant intelligence, sensibilité, aptitudes manuelles, physiques et artistiques de l'apprenant. Dans l'enseignement moyen, il vise la maîtrise, pour chaque élève, d'un socle de compétences incompressibles d'éducation, de culture et de qualification lui permettant de poursuivre des études et des formations post-obligatoires ou de s'intégrer dans la vie active. Les champs disciplinaires sont marqués par les transversalités entre programmes.

\section{Les langues}

Langue arabe: symbole de la souveraineté nationale, instrument de communication transdisciplinaire par excellence, sa maîtrise est déterminante pour la mise en place des apprentissages afin que l'élève parvienne à un niveau optimal d'assimilation, de compréhension et d'utilisation du savoir tant à l'oral qu'à l'écrit. L'amélioration de son enseignement est un objectif majeur de la refonte pédagogique, afin de lui donner sa pleine fonction pédagogique et socio culturelle.

Langue tamazight: langue nationale officielle depuis sa constitutionnalisation en mars 2016 (enseignée dans 11 wilayas ${ }^{8}$ sur 48), sa généralisation est à l'ordre du jour. Introduite en quatrième année du primaire, sa standardisation, par l'unification de sa transcription, est attendue dans les manuels scolaires.

Langues étrangères: le français, première langue étrangère en troisième année du primaire, connaissant un déficit important d'enseignants, notamment dans le sud du pays; et l'anglais, deuxième langue étrangère dès la première année du moyen, sont enseignées en tant qu'outil de communication et d'ouverture sur le monde.

7. Pour le premier palier du primaire ( $1^{\text {re }}$ et $2^{\mathrm{e}}$ années) et du moyen ( $1^{\text {re }}$ année).

8. Wilaya : département. 


\section{Les sciences et la technologie}

Les mathématiques : améliorer les résultats (jusqu'à l'excellence) et mieux former les enseignants sont les objectifs au vu des résultats au BEM. ${ }^{9}$ L'utilisation de la symbolique universelle a déjà donné des résultats positifs.

Sciences physiques et technologiques: l'éducation technologique est au primaire une nouvelle discipline. Initiés dès la première année du primaire, les élèves construisent progressivement des connaissances pour développer des compétences scientifiques et technologiques associées à des démarches.

Sciences de la nature et de la vie: apprentissages organisés en domaines destinés à sensibiliser l'élève à la préservation de sa santé et de son environnement.

Les matières scientifiques, avec un coefficient élevé, sont perçues comme valorisantes et essentielles pour la réussite au BEM.

\section{Les sciences sociales et humaines}

Histoire et géographie : sont introduites en troisième année du primaire : ancrage des valeurs identitaires par le développement du sentiment d'appartenance à un pays, une nation, une civilisation, installation des premières connaissances du patrimoine historique et culturel du pays; structuration de l'espace et du temps vécus. Les programmes d'histoire, dans les différents paliers, accordent à l'histoire nationale une place prépondérante. L'apprenant, imprégné de son passé national, dans tous ses aspects, doit s'intéresser à l'histoire du monde et de ses civilisations.

Éducation islamique : enseignée de la première année du primaire à la terminale, elle a pour objectifs de développer, en plus de l'apprentissage du Coran, l'acquisition de comportements individuels et collectifs conformes aux nobles valeurs de l'islam (sens de la justice, solidarité, travail, tolérance...).

L'éducation civique : l'acquisition de savoirs sur la citoyenneté, les droits de l'homme, la démocratie, le fonctionnement des institutions politiques et sociales, le développement durable, etc., doit se traduire en comportements développant le sens des responsabilités vis-à-vis de la société et du bien commun.

L'histoire, l'éducation islamique ainsi que la langue arabe, étroitement liées au socle identitaire sont des disciplines qualifiées de "fil rouge " par des associations, syndicats et autres partenaires de l'éducation. De vifs débats s'engagent dès qu'elles font l'objet de propositions d'aménagements.

\section{L'éducation artistique et sportive}

Musique et dessin : disciplines longtemps négligées ou volontairement écartées faute d'encadrement, elles sont enseignées au collège (ou l'une ou l'autre).

9. «En 2012, ce sont plus de $55 \%$ des candidats qui obtiennent des notes inférieures à 10/20 », Revue Éduc/ Recherche, dossier « Les mathématiques », 2014, $\mathrm{n}^{\circ}$ 7, p. 28. 
Éducation physique et sportive: son caractère obligatoire est valable de la première année à la sortie de l'enseignement secondaire.

Les disciplines artistique et sportive sont en cours de réhabilitation à l'école.

\section{Les TICE}

Informatique : enseignée dans le moyen, cette discipline ne fait pas l'objet d'une évaluation au BEM. Sa généralisation, conformément au plan national d'informatisation, est en cours en vue de donner aux élèves une culture informatique de base. Par ailleurs, en 2015, l'indicateur REO (nombre moyen d'élèves par ordinateur, par niveau et par année scolaire) affichait 669 élèves par ordinateur pour le primaire et 58 pour le moyen. Le degré d'intégration de l'outil informatique n'est pas du niveau d'exigence requis par et pour notre époque.

La multiplication des disciplines pose le problème de la gestion rationnelle du temps scolaire. Pour éviter l'émiettement du savoir et les usages répétitifs, les nouveaux programmes sont établis pour 32 semaines d'enseignement (au lieu de 28) mais restent encore en deçà des standards internationaux en la matière.

\section{Certification des compétences de l'enseignement obligatoire}

Le cycle de l'enseignement moyen est sanctionné par un examen national ouvrant droit à l'obtention du BEM. Ce diplôme permet l'accès à l'enseignement secondaire général et technologique ou à l'enseignement professionnel, ou encore à la formation professionnelle. Les élèves évalués, à l'écrit, dans neuf disciplines, dont le plus fort coefficient est celui de la langue arabe, doivent obtenir une moyenne finale de 10/20, pour être admis au lycée. En première année secondaire, le tronc commun des sciences et technologie connaît un attrait particulier, hormis ses filières mathématiques, en forte diminution d'orientation. Les élèves ayant obtenu les meilleures moyennes au BEM et 20/20 en maths sont orientés vers l'unique lycée national de mathématiques (ouvert en 2012), à raison de deux ou trois élèves par wilaya.

À la session BEM 2016, le taux de réussite au niveau national est de 54,42\% pour un total de 546076 candidats, un résultat éloigné de l'objectif « d'amélioration des taux de succès aux examens scolaires dans des proportions significatives : entre $70 \%$ et $80 \%$ » prévu par la Loi d'orientation. Les résultats sont jugés peu satisfaisants en français, anglais et mathématiques, avec toutefois, une « légère amélioration dans la moyenne des mathématiques qui a atteint 9,06/20 contre 7,67/20 en $2015 »{ }^{10}$ Ce bilan peu probant est encore plus mitigé quand on sait que seul un tiers des élèves se présentant au BEM a l'âge scolaire attendu, ce qui signifie que la majorité des élèves a été amenée à redoubler (Éduc/Recherche, 2016).

À chaque fin d'année scolaire, de nombreux recours témoignent de failles dans le système d'orientation. Un niveau de quatrième année du moyen ne

10. Conférence de presse du 17 juin 2016, Inspection générale, Ministère de l'éducation nationale, Alger. 
débouche sur aucune qualification précise. L'articulation entre l'éducation nationale et la formation professionnelle n'est pas régulée en termes d'orientation. La formation et l'enseignement professionnels restent peu attractifs, et sont souvent perçus comme réceptacle de l'échec scolaire. Ceci nous éloigne de l'objectif visant à donner aux élèves « les éléments pour choisir une orientation post-obligatoire, favoriser l'ouverture de l'école vers l'environnement socioculturel, technologique, professionnel» (Commission nationale des programmes, 2009).

De plus, le dispositif permanent de suivi et d'évaluation des acquis scolaires (créé en 2002) pour le suivi de la qualité de l'enseignement dans les cycles primaire et moyen, en langue arabe, langues étrangères, mathématiques et sciences révèle, dans l'évaluation des résultats de quatrième année du moyen (2011), des pourcentages importants d'élèves en difficulté : $66 \%$ en français, $59,3 \%$ en mathématiques, $48 \%$ en physique, $32 \%$ en anglais, $17 \%$ en langue arabe, 8,4 \% en sciences naturelles (Mezaïb, 2015).

Les signes les plus visibles des maux qui hypothèquent la politique de scolarisation obligatoire sont les résultats insuffisants au BEM, mais aussi les résultats inégaux par wilaya et par établissement, qui amènent à s'interroger sur les ressources déployées et sur leur utilisation ${ }^{11}$. Forte préoccupation actuelle, la déperdition scolaire (taux de redoublement de 11,86\% pour l'enseignement primaire et moyen et taux d'abandons de $4,77 \%{ }^{12}$ ) est due notamment à des modalités d'évaluation peu appropriées et à une formation déficiente des enseignants.

En conclusion, la volonté de l'Algérie de donner à tous un enseignement obligatoire, commun dans son essence et sa philosophie, dans un esprit d'équité, d'égalité des chances et de justice, consacrée juridiquement par l'adhésion à nombre de résolutions sur l'éducation adoptées par les instances internationales, ainsi que par les multiples articles de la Loi d'orientation, ne connaît pas encore d'aboutissement à hauteur des ambitions de la réforme. Celle-ci prévoyait « l'homogénéisation et l'amélioration des paramètres de scolarisation à l'intérieur d'une même wilaya et entre les wilayas du territoire, la réduction significative des déperditions en cours de scolarité de manière à faire parvenir, d'ici à 2015, $90 \%$ d'une cohorte de première année primaire en quatrième année moyenne ${ }^{13}$ ». Si les objectifs en matière d'amélioration des résultats de l'enseignement obligatoire ne sont pas encore atteints au vu des normes du référentiel algérien, la fréquence des redoublements et des abandons affectant sensiblement l'efficacité de cet enseignement, des mesures d'amélioration des résultats ont déjà été prises sur le terrain.

11. Mingat Alain (2015) : "Évaluer les acquis et les processus éducatifs : prendre en compte les lieux et le temps », communication, colloque international « Évaluation des acquis des élèves : état des lieux et perspectives », 3-4 juin 2015, INRE, Alger.

12. Enquête statistique, 2014, MEN, Sous-direction des statistiques, Alger.

13. Préambule de la Loi d'orientation sur l'éducation nationale (2008). 
Pour les renforcer et repousser les limites du «lire, écrire, compter et cliquer» de l'école algérienne, on pourrait développer les compétences professionnelles des enseignants à mettre en œuvre de nouveaux programmes et mettre en place un dispositif d'évaluation essentiellement formative qui accompagnerait les programmes de deuxième génération. La pédagogie de la remédiation, institutionnalisée tout au long de l'enseignement obligatoire, endiguerait les déperditions scolaires. De plus, il serait intéressant d'encourager l'exploitation des ressources locales et spécificités régionales (patrimoine, pédagogie muséale, etc.), pour donner du sens à ce que l'école enseigne dans le cadre du projet d'établissement, promu depuis quelques années. D'autre part, pour une plus grande équité, la révision du système national d'orientation afin d'accéder à une formation s'impose, de même que la recherche d'équilibre en matière de distribution des ressources par /établissement.

Encourager la participation de l'Algérie aux évaluations internationales (première participation de l'Algérie à TIMSS en 2007 et à PISA en 2015), avec publication et diffusion des résultats, permettrait aussi de mieux prendre la mesure de ce qu'on enseigne. L'amélioration du système d'information statistique contribuerait, enfin, à donner une meilleure représentativité du système scolaire algérien. Il est encourageant de constater qu'en ces temps de crise économique, l'Algérie continue à considérer le secteur éducatif comme prioritaire puisqu'il ne connaît pas de restriction budgétaire. La rentrée 2016 a enregistré 28000 postes pédagogiques supplémentaires. La maîtrise des apprentissages fondamentaux est aussi à ce prix.

\section{Bibliographie}

LAKHDAR B. (2016) : «Le redoublement et ses effets néfastes sur le parcours scolaire des élèves ", Revue Éduc /Recherche $\mathrm{n}^{\circ}$ 14, Alger.

MEN (2010) : «Évaluation de la mise en œuvre de la réforme du système éducatif par le biais d'indicateurs, plan de développement 2010-2014 », rapport, août, Alger : MEN. MEN/Commission nationale des programmes (2009): Référentiel Général des Programmes, Alger : MEN.

MEN/INRE (1999): Mémorandum: évaluation des programmes de l'enseignement fondamental et secondaire, MEN/INRE, Alger.

MEN/Programme d'appui de l'UNESCO à la réforme du système éducatif PARE (2005) : La Refonte de la pédagogie en Algérie, août, Bureau de l'Unesco pour le Maghreb/ Rabat [en ligne] [goo.gl/SDHw5D].

MEN / INRE / UNICEF (2012) : Élaboration d'un référentiel d'indicateurs d'évaluation des normes assignées au système scolaire algérien, Alger.

MEN/Sous-direction des statistiques (2015) : Enquête statistique, 2014-2015, MEN, Alger. MEZAIB S. (2015) : «L'évaluation des acquis scolaires en Algérie, approche nationale et internationale », Conférence nationale, juillet, Palais des nations, Alger : INRE.

Revue Éduc/Recherche (2014): dossier "Les mathématiques», n 7, Alger: INRE. [en ligne] [https://goo.gl/nbF8Oc]

Revue Éduc/Recherche (2016) : dossier "Des pistes pour lutter contre le décrochage scolaire ", $\mathrm{n}^{\circ} 14$, Alger : INRE. [en ligne] [https://goo.gl/DrgEiW] 